\title{
Affective and Behavioural Dysfunctions in Patients with Subcortical Stroke
}

\author{
Luis Gutierrez Cabello ${ }^{1}$, Isabel Gómez-Ruiz ${ }^{2}$, Angel Aguilar Alonso ${ }^{3}$, Salvador Pedraza Gutiérrez ${ }^{4}$ \\ ${ }^{1}$ Department of Basic Psychology, University of Barcelona, Barcelona, Spain; ${ }^{2}$ Department of Neurology, Hospital General de \\ L'Hospitalet, Hospitalet de Llobregat, University of Barcelona, Barcelona, Spain; ${ }^{3}$ Department of Personality, Psychological As- \\ sessment and Treatment, University of Barcelona, Barcelona, Spain; ${ }^{4}$ (IDI) Service of Radiology, Dr Josep Trueta University Hospi- \\ tal, Girona, Spain. \\ Email: gutierrezlgcabe@ub.edu,spedraza@retamail.es
}

Received February $9^{\text {th }}, 2012$; revised March $14^{\text {th }}, 2012$; accepted April $15^{\text {th }}, 2012$

\begin{abstract}
Objective: To investigate the association of ischaemic and haemorrhagic strokes with the site of subcortical focal cerebrovascular injuries with affective, behavioural and cognitive dysfunctions in the acute phase and after three months. Sample: 58 patients with focal cerebrovascular injuries; mean age $61.5 \pm 13.5,72.4 \%$ male. Control group: 20 healthy subjects, mean age $67.1 \pm 7.6,50 \%$ male. Results: Significant differences were observed $(p=0.006)$ between the acute phase and three months later on the apathy items of the Neuropsychiatric Inventory (NPI) in patients with subcortical stroke. Significant differences were also observed on the affective scale of the BRMS $(p=0.004)$, the behaviour scale of the Maudsley Obsessive-Compulsive Inventory $(\mathrm{MOCI})(\mathrm{p}=0.000)$, and on the Mini Mental State Examination $(\mathrm{MMSE})(\mathrm{p}=0.006)$. No significant differences were observed on the Hamilton Rating Scale-Depression (HRSM) $(\mathrm{p}=$ 0.101). Significant differences were found according to the infarct type: patients with haemorrhagic lesions had higher raw scores on the HRS-D than those with ischaemic lesions $(p=0.024)$. Conclusions: The performance of patients with subcortical stroke on affective, behavioural and cognitive scales improves after three months. Patients with haemorrhagic lesions are more likely to be depressive than those with ischaemic lesions.
\end{abstract}

Keywords: Subcortical Lesions; Stroke; Cognitive; Affective and Behaviour

\section{Introduction}

A wide variety of neuropsychiatric syndromes are associated with right hemisphere brain lesions: mania, psychosis, hallucinations, personality changes, anxiety, dissociative states and alterations of sexual behaviour [1]. It is also said that many neuropsychiatric syndromes are the consequence of alterations in the limbic system [2]. Specifically, some authors [3] state that focal cerebrovascular injuries may be followed by Kraepelin's major depressive disorder. In one study the prevalence [4] of postinfarct depression varied from $25 \%$ to $50 \%$ during the recuperation phase and in another [5] between $18 \%$ and $78 \%$ in the first two years, reaching its highest point between 3 and 6 months after stroke. Other authors $[6,7]$ observed hypomanic episodes in patients with thalamic lesions of vascular origin in the right hemisphere. Starkstein et al. [8] suggested that lesions in the basotemporal lobe and orbitofrontal cortical region lead to a syndrome of disinhibition, secondary to a manic process. It has also been noted that the integrity of the frontolimbic and basal ganglia connections is necessary for a "normal mood" [9].
Furthermore, patients with focal cerebrovascular lesions in frontal regions and basal ganglia are susceptible to obsessive-compulsive disorders due to the dysfunction of frontal subcortical circuits [10-12]. This study included patients with lesions in the mesencephalon, the protuberance cerebral peduncle and the cerebellum given that there are cortical-thalamus-ponto-cerebellum connections that can cause motor, affective and cognitive impairment [13].

The aim of the present study is to relate possible affective, behavioural and cognitive changes with the location and type of subcortical focal cerebrovascular injuries, in the acute phase and after three months.

\section{Material and Method}

\subsection{Sample}

The sample comprised of 58 patients with focal cerebrovascular lesions in subcortical structures: $72.4 \%(\mathrm{n}=$ 42) were male and $27.6 \%(n=16)$ female, with a mean age of $(61.5 \pm 13.5)$ years, and a mean educational level 
of $(6.9 \pm 4.2)$ years. Patients were admitted to the Neurology Service of the Dr. Josep Trueta Hospital of Girona. We also assessed a control group from the Santa Creu and Sant Pau Hospital of Barcelona, comprising 20 healthy subjects, $50 \%$ male and $50 \%$ female, with a mean age of $(67.1 \pm 7.6)$ years and mean educational level of $(7.6 \pm 3)$ years. Written informed consent was received from all subjects. The mean period between admission to the neurology service and the administration of the neuropsychological tests and scales was $(6.2 \pm 7.2)$ days. The second evaluation was performed in 44 patients of the total sample after approximately three months. Patients were admitted to the study if lesions in the cerebral structures (basal ganglia, corona radiata, thalamus, mesencephalon, protuberance cerebral peduncle and cerebellum) in the MRI and CT were observed by a radiologist blinded to the clinical data of the study. Exclusion criteria: Patients with severe comprehension problems and those who had a previous history of mental illness as defined by the DSM-IV-TR criteria for mental disorders [14] were excluded.

\subsection{Tests Administered}

- Neuropsychiatric Inventory (NPI) [15]. This global symptoms scale was administered to patients to obtain a profile of possible behaviour disorders. Information was gathered on the presence of mental illness in patients with brain disorders. The scale comprises 11 items: delusions, hallucinations, agitation, depresssion, anxiety, euphoria, apathy, indifference, disinhibition, irritability and motor disturbance. The frequency is scored on a scale from 1 (occasionally) to 4 (very frequently) and severity is assessed according to three levels (mild, moderate or high).

- Hamilton Rating Scale for Depression (HRS-D) [16]. This scale was used to observe possible affective disorders and to assess the patients' mood post-stroke. It comprises 10 items and evaluates the following aspects: depressed mood, feelings of guilt, suicide attempts, insomnia, work and activities, slowness of thought, agitation, psychological and somatic anxiety, somatic symptoms, genital symptoms, hypochondriasis, insight and weight loss.

- Bech-Rafaelsen Mania Scale (BRMS) [17]. This scale detects hypomanic processes. It evaluates motor activity, flight of thoughts and ideas, loudness of voice and noisiness, mood and feeling, self-esteem, verbal contact, sleep, sexual activity and work and interests.

- Maudsley Obsessive Compulsive Inventory (MOCI) [18]. This scale comprises four sub-scales with a total of 30 items that assess checking, cleaning, slowness and doubting.

- Folstein's Mini Mental Examination State (MMSE)
[19]. This scale evaluates the global cognitive performance; it comprises 30 items assessing temporalspatial orientation, memory, attention and calculation, naming, repetition, reading, writing and drawing.

\subsection{Neuroimaging Equipment}

- Magnetic resonance imaging (MRI) of the Doctor Josep Trueta University Hospital (1.5 Teslas Philips, Giroscan). The sequences used were:

DWI (Diffusion-weighted imaging), PWI (Perfusion weighted imaging),

FLAIR (fluid attenuated inversion recovery). T2 weighted.

- Computerized axial tomography imaging (CAT) of the Doctor Josep Trueta University Hospital. Philips medical Systems MX 8000-D, 2 slice acquisition.

- CT Unit Picker 5000. Cranial Study of 4 and $8 \mathrm{~m}$ slices with FOV of 240.

\subsection{Procedure}

Administration of cognitive and affective scales:

The cognitive global tests (MMSE) and affective, cognitive-behaviour scales were administered in the first week after stroke, and a second evaluation was carried out after approximately three months.

\subsection{Neuroimaging Analysis}

All patients received a high precision cranial CT on admission in order to rule out haemorrhage. In addition, $71 \%(n=41)$ of the sample received MRI to locate the lesion according to neurological symptoms. To locate the lesion in the subcortical structures, a neuroimaging atlas was used (the Spanish version of the Atlas of Anatomic Correlations in CT and MRI) [20]. Stroke was ischaemic in $82.7 \%$ of the sample $(\mathrm{n}=48)$ and haemorrhagic in $17.24 \%(n=10)$. The lesions were left-sided in $46.5 \%$ of subjects $(n=27)$, right-sided in $43.1 \%(n=25)$, and bilateral in $10.3 \%(\mathrm{n}=6)$. The stroke had occurred in the corona radiata in $25.9 \%(\mathrm{n}=15)$, in the basal ganglia and thalamus in $53.4 \%(\mathrm{n}=31)$, in the mesencephalon and protuberance in $13.8 \%(\mathrm{n}=8)$; and in the cerebellum in $6.9 \%(n=4)$. Recurrent lesions were observed in $21.8 \%$ patients $(n=17)$ of the sample (See Table 1).

\subsection{Statistical Analysis}

To verify the significance of the differences between the means of the samples, the following statistical tests were performed: the Student " $\mathrm{t}$ " test for independent samples and the Student " $t$ " test for related samples, with a confidence interval of $95 \%$. The chi-square test was used to evaluate the sex variable.

PASW Statistics 18 for Windows was used for data analysis. 
Table 1. Characteristics of the lesion site.

\begin{tabular}{ccccccc}
\hline & Corona radiata & $\begin{array}{c}\text { Basal Ganglia and } \\
\text { Thalamus }\end{array}$ & $\begin{array}{c}\text { Mesencephalon and Protuberance } \\
\text { Cerebral Peduncle }\end{array}$ & Cerebellum & Total \\
\hline \multirow{3}{*}{ Lesion } & Right & 6 & 16 & 5 & 0 & 27 \\
& Left & 6 & 13 & 3 & 3 & 25 \\
& Bilateral & 3 & 2 & 0 & 4 & 58 \\
\hline
\end{tabular}

\section{Results}

\subsection{Comparison of Patients with Subcortical Stroke and Healthy Subjects}

The characteristics of the study groups are presented in Table 2. With regards to the demographic variables, the study group was found to differ significantly in terms of age but not with regards to educational level and sex.

Patients with subcortical stroke are found to obtain higher mean scores on the HRS-D than controls, although there was no difference between the groups according to statistical analysis $(p=0.184)$. However, on the global cognitive performance scale MMSE, the performance of patients was worse than of the controls $(\mathrm{p} \geq$ $0.000)$.

In the pathological group we found that $20 \%$ of patients had white matter and paraventricular lesions and $8.6 \%$ had cerebral atrophy.

\subsection{Differences between the Acute Phase and Three Months Post-Injury}

None of the patients with focal cerebrovascular injuries in subcortical structures responded positively on the delusions, euphoria and motor disturbance items in the acute phase. In contrast, some responded affirmatively on the hallucinations, agitation, depression, anxiety, apathy, disinhibition and irritability items both in the acute phase and after three months. Table 3 shows the numbers of subjects who were positive for the various items on the Neuropsychiatric Inventory, the means of their raw scores and the range of scores (minimum to maximum) in the acute phase and after three months. This table shows that few patients had clear evidence of behaviour disorders due to subcortical vascular lesions. As we noted above, none of the patients were positive for the delusion item either in the acute phase or after three months. On the hallucinations and agitation items only one patient responded affirmatively in the acute phase, while after three months none responded affirmatively for hallucinations, although two were positive for agitation. Interestingly, ten patients responded affirmatively on depression in the acute phase, and only six after three months. In the acute phase none of the patients responded affirmatively for euphoria, and one did so after three months. On the anxiety item four responded affirmatively in the acute phase, and five after three months. On apathy seven subjects responded affirmatively in the acute phase, and only five after three months. For disinhibited behaviour, five patients responded affirmatively in the acute phase, and only one after three months. On irritability five responded in the acute phase, and two after three months. Finally, no patients responded affirmatively on motor disturbance either in the acute phase or after three months.

Table 4 compares the mean raw scores on the HRS-D in the acute phase and after three months. The data show an improvement in the depressive symptoms in the second evaluation, although there was no difference according to statistical analysis $(p=0.007)$. In contrast, statistically significant differences were found between the acute phase and after three months on the BRMS ( $p=$ $0.004)$, on the MOCI $(p=0.000)$ and on global cognitive performance measured with the MMSE $(p=0.006)$.

Using the HRS-D standard cut-off point $\geq 9$ for distinguishing between mild and moderate depression (Hamilton, 1960) nine patients (20.7\%) had clear symptoms of depression in the acute phase, but only four after three months.

\subsection{Results According to Lesion Site}

The means of the affective, cognitive behavioural and cognitive tests by the side of the lesion were compared. A multivariant test was performed. Firstly, the tests of the equality of the covariant matrixes ( $\mathrm{M}$ de Box) $(\mathrm{p}=$ $0.023)$. There were significant differences between the variance of the groups studied. Application of the Levene Contrast Test revealed the following: HRS-D ( $p=0.355)$; BRMS $(p=0.171) ; \operatorname{MOCI}(p=0.002) ;$ and MMSE $(p=$ 0.495). A statistically significant difference in the MOCI groups are compared. No statistically significant differences are observed when the side of the lesion is compared using the different scales: HRSD (F: $1.320 \mathrm{p}=$ 0.256); BRMS (F: $1.458 \mathrm{p}=0.233$ ); MOCI (F: $0.235 \mathrm{p}=$ 0.630 ); and MMSE (F: $1.416 \mathrm{p}=0.240)$. The power calculations to reveal the influence of lesion side on the emotional symptoms were: HRS-D (0.203); BRMS (0.220); 
Table 2. Characteristics of the study groups.

\begin{tabular}{|c|c|c|c|c|}
\hline & Pathological Group $(\mathrm{n}=58)$ & Control Group $(\mathrm{n}=20)$ & Statistics & $\mathrm{p}$ value \\
\hline Age & $61.3(13.4)$ & $67.2(7.4)$ & F: 16.11 & 0.015 \\
\hline Education, years & $6.9(4.2)$ & $7.4(3.1)$ & F: 1.4 & 0.247 \\
\hline Sex & $42 / 16$ & $10 / 10$ & $X^{2}: 3.4$ & 0.067 \\
\hline Depression (HRS-D) & $4.7(3.9)$ & $3.6(3.4)$ & F: 1.8 & 0.184 \\
\hline MMSE $^{*}$ & $26.6(2.7)$ & $29.3(0.7)$ & F: 14.4 & $>0.000$ \\
\hline \multicolumn{5}{|l|}{ MRI findings } \\
\hline Ischaemic & $82.7 \%(\mathrm{n}=48)$ & & & \\
\hline Haemorrhagic & $17.24 \%(\mathrm{n}=10)$ & & & \\
\hline DWMHs $^{\dagger}$ & $20 \%$ & & & \\
\hline Atrophy & $8.6 \%$ & & & \\
\hline Recurrent les. & $21.8 \%$ & & & \\
\hline
\end{tabular}

"MMSE: Mini Mental State Examination; 'DWMHs: Deep White Matter Hyperintensitivites Paraventricular.

Table 3. Frequency and means of the raw scores of the patient group in the Neuropsychiatric inventory: acute phase and at three months.

\begin{tabular}{|c|c|c|c|c|c|c|}
\hline & Acute phase & $\mathrm{M} \pm \mathrm{SD}$ & Range & Three months & $\mathrm{M} \pm \mathrm{SD}$ & Range \\
\hline Delusions & - & - & - & - & - & - \\
\hline Hallucinations & 1 & 1 & $(1-1)$ & - & - & - \\
\hline Agitation & 1 & 1 & $(1-1)$ & 2 & $1.5 \pm 0.7$ & $(1-2)$ \\
\hline Depressed mood & 10 & $2.2 \pm 1.5$ & $(1-6)$ & 6 & $2.6 \pm 1.2$ & $(1-4)$ \\
\hline Euphoria & - & - & - & 1 & $6 \pm 0$ & $(6-6)$ \\
\hline Anxiety & 4 & $2.7 \pm 2.3$ & $(1-6)$ & 5 & $2 \pm 0$ & $(2-2)$ \\
\hline Apathy & 7 & $2.0 \pm 0.5$ & $(1-3)$ & 5 & $2.8 \pm 2.9$ & $(1-8)$ \\
\hline Disinhibition & 5 & $4 \pm 4.4$ & $(2-12)$ & 1 & $6 \pm 0$ & $(6-6)$ \\
\hline Irritability & 5 & $2.2 \pm 2.1$ & $(1-6)$ & 2 & $2.5 \pm 0.7$ & $(2-3)$ \\
\hline Motor disturbance & - & - & - & - & - & - \\
\hline
\end{tabular}

Table 4. Means of the raw scores and SD of the different scales of the patient group in the acute phase and at three months.

\begin{tabular}{ccccc}
\hline Scales & (n) & Acute phase Means (SD) & 3 months Means (SD) & $\mathrm{p}$ value \\
\hline HRS-D & 44 & $4.8 \pm(4)$ & $2.8 \pm(3.5)$ & 0.101 \\
BRMS & 44 & $2.07 \pm(3)$ & $0.84 \pm(1.5)$ & 0.004 \\
MOCI & 40 & $13.1 \pm(4.6)$ & $10 \pm(4.4)$ & $>0.000$ \\
MMSE & 44 & $26.7 \pm(2.8)$ & $27.9 \pm(2.1)$ & 0.006 \\
\hline
\end{tabular}

(HRS-D) Hamilton Rating Scale Depression; (BRMS) Beck Rafaelsen Mania Scale, (MOCI) Maudsley Obsessive-compulsive Inventory; (MMSE) Mini Mental State Examination. 
MOCI (0.076); MMSE (0.215). The small size of the sample and low scores obtained for the power calculations in the different variables make it clear that studies with larger and more homogeneous samples will be required in order to confirm whether or not emotional state is more associated to one hemisphere or another (Table $5)$.

\subsection{Comparison According to Lesion Type}

Three cut-off points were applied to the Hamilton Depression Scale (HRS-D). Group I, patients with depressive symptoms of between 8 and 16 , consisted of $25.9 \%$ in the acute phase. Group II, patients with depressive symptoms of between 1 and 7 , consisted of $55.2 \%$. Group III, patients without depressive symptoms, consisted of $19 \%$. At three months the proportions of patients in each group were: Group I, 8.6\%; Group II, $36.2 \%$; and Group III, $55 \%$. Only $15.9 \%(\mathrm{n}=9)$ of patients received pharmacological treatment for depression.

Univariate analysis (ANOVA) was performed taking the direct scores on the HRS-D depression scale as dependent variables and the type of infarct (ischaemic/ haemorrhagic) and the lesion site (left/right/bilateral) as factors. The type of infarct had a statistically significant effect ( $p=0.024$; Figure 1) with haemorrhagic infarct patients presenting higher mean scores than those with ischaemic infarct.

\section{Discussion}

The comparison of mean scores for global cognitive and affective performance in patients with subcortical stroke and controls resulted in clear differences being found. Subcortical stroke patients showed a degree of cognitive deterioration, although the difference was not statistically significant. Previous studies have associated major depression with cortical lesions in the area of the frontal pole and the basal ganglia of the left hemisphere [21,22].

After the second evaluation performed at three months, patients with subcortical stroke $(n=44)$ presented a nonsignificant reduction in their depressive symptoms, and a

Table 5. Means of raw scores on the scales and differences between patients with subcortical lesions in right and left hemispheres.

\begin{tabular}{ccccccc}
\hline Scales & $(\mathrm{n})$ & LH Means (SD) & RH Means (SD) & F & Power observed & p value \\
\hline HRS-D & $27 / 25$ & $5.4 \pm(4.3)$ & $4.1 \pm 3.7$ & 1.32 & 0.203 & 0.256 \\
BRMS & $27 / 25$ & $1.4 \pm(2.1)$ & $2.3 \pm(3.3)$ & 1.45 & 0.220 & 0.233 \\
MOCI & $26 / 24$ & $12.1 \pm(6.1)$ & $12.8 \pm(3.8)$ & 0.235 & .076 & 0.630 \\
MMSE & $27 / 25$ & $26.4 \pm(2.3)$ & $27.2 \pm(2.5)$ & 1.46 & 0.215 & 0.240 \\
\hline
\end{tabular}

(HRS-D) Hamilton Rating Scale Depression; (BRMS) Beck Rafaelsen Mania Scale; (MOCI) Maudsley Obsessive-compulsive Inventory; (MMSE) Mini Mental State Examination.

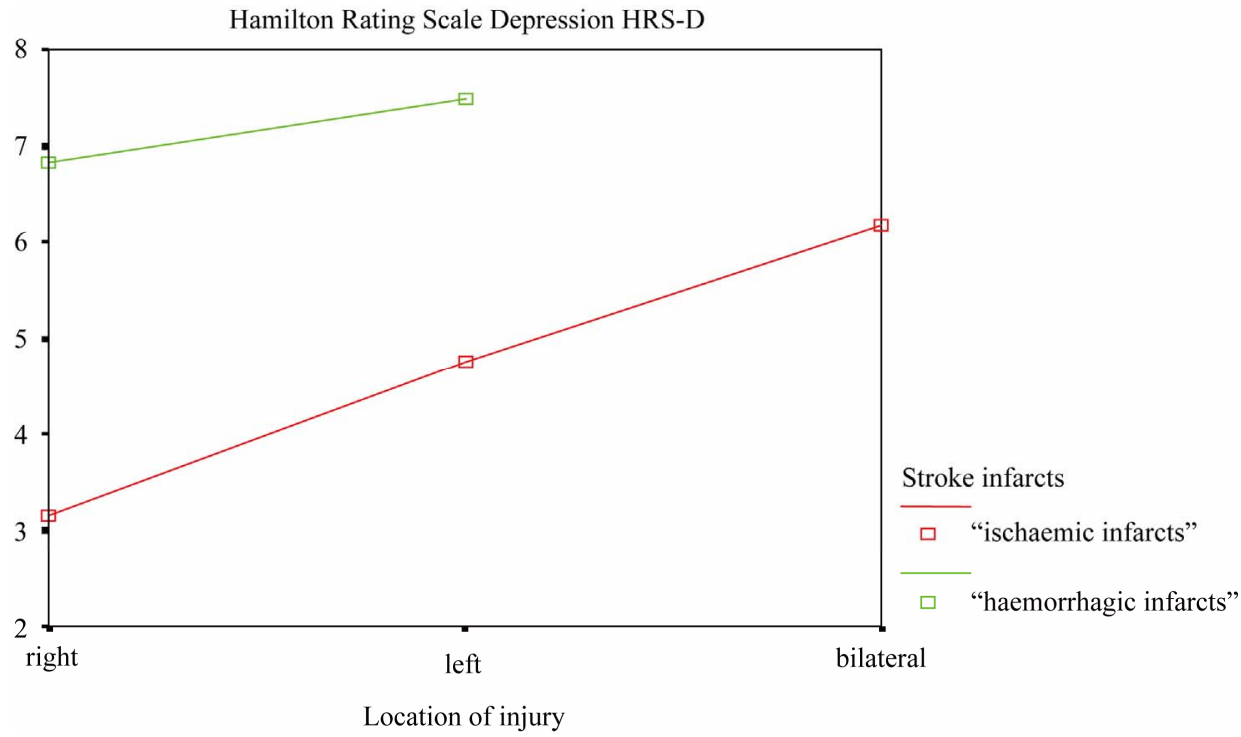

Figure 1. Relation between HRS-D scores, type of infarct (ischaemic/haemorrhagic), and the hemisphere involved (left/right) in the stroke group patients. 
clear, significant improvement in the affective and behavioural scales (BRMS and MOCI). There was also a clear improvement in global cognitive performance measured using the MMSE.

No significant differences were found between lesion site (left/right) and the mean scores on the affective scales, but patients with left-sided lesions scored higher on the HRS-D, and patients with right-sided lesions scored higher on the BMRS though neither of the differences reached statistical significance. In general, studies published to date on the association of lesion site and affective disorders are highly controversial. In 15 patients with lesions due to cerebrovascular accident (CVA) and cranioencephalic traumatisms, Lipsey et al. [23,24] found that nine presented major depression according to DSMIV criteria. In another study in stroke patients, Robinson [25] observed that major depression appeared more frequently in those with lesions in the left frontal lobe. However, House et al. [26] replicated these studies and found no association between major depression and left hemisphere lesions. Signer et al. [27] observed that anterior right hemisphere lesions were associated more with depressive disorders than posterior right lesions, and that euphoria was more frequent in posterior right lesions in 61 patients in the sample. For their part, Stern and Bachman [28] found that patients with frontal lobe and right superior temporal lesions frequently have depressive symptoms post-stroke.

Other authors [29] have associated right hemisphere lesions with hypomanic processes, arguing that an interaction between injury to certain areas of the right hemisphere lesion and genetic factors may produce secondary mania. In our sample, the right-sided lesion patients scored higher on the Bech-Rafaelsen Mania Scale (BRMS) than those with left-sided lesions, though as we noted above the difference was not statistically significant.

On the Maudsley Obsessive-Compulsive Inventory (MOCI), no significant differences were observed according to the site of lesion in our sample. However, Simpson and Baldwin [30] described a patient with no previous psychiatric history who developed an obsessive-compulsive disorder associated with a right inferior parietal infarct and decreased regional cerebral blood flow revealed by SPECT in the right basal ganglia and temporal areas.

On the Neuropsychiatric Inventory (NPI), some patients presented depression, anxiety, apathy, irritability and disinhibition, both in the acute phase and after three months. Several authors have reported apathy, euphoria, irritability and lability in patients with vascular dementia. [31] However, in our sample of patients with subcortical focal vascular brain lesions we did not observe behavioural symptoms, euphoria, motor disturbance or personality changes. One patient presented hallucinations and agitation, symptoms that appear more typically in degenerative processes such as Alzheimer's disease [32].

The findings in preliminary studies of associations between left anterior lesions and depressive states have also caused controversy. For example, it has been said that major depression may be overdiagnosed in CVA patients, and that it may in fact be a consequence of the changes in sleeping patterns, loss of appetite, loss of sex drive, and so on, as a result of the medical illness [33]. Our patients with subcortical vascular lesions presented anxiety, apathy, irritability, disinhibition, and depressive symptoms both in the acute phase and after three months. To a lesser extent we observed hallucinations and agitation, but we did not find delusion or motor disturbance. We believe that these behaviours may be the result of the lesions produced by the stroke, causing fronto-thalamocortical disconnections.

Hackett et al. (2005) observed a prevalence of major depression in the acute phase of $33 \%$, which remained similar in the medium and long term. However, in our study we find a similar prevalence of depression in the acute phase $(25.9 \%)$ but a significant reduction in prevalence at three months $(8.6 \%)$. The explanation for this difference may well be found in the widely varying diagnostic tools used in the different studies analysed by Hackett, many of which, for example, excluded patients with cognitive impairment, patients with aphasia, and patients with a previous history of depression. Data was gathered by means ranging from self-administered questionnaires to structured interviews with trained health professionals. Another difficulty in making comparison with Hackett is, as the authors recognise, that some of the studies included fail to take into account the initiation of pharmacological treatment nor distinguish between depressive symptoms in the acute phase and reactive depression.

Comparing our patients with the healthy subjects, we found that in the acute phase subcortical stroke sufferers had poorer cognitive performance than controls. This finding suggests a role for subcortical structures in cognition, via the cortical-subcortical connections; the dysfunction may be due to diaschisis, or alternatively because in the acute phase the lesion affects not only its structural area, but also an area of dense schaemia involving cortical areas [34].

We also observed that in the acute phase of stroke patients with subcortical lesions scored higher on obsessive-compulsive behaviours, mania, depression and lower on global cognitive performance than after three months. Patients with haemorrhagic lesions scored higher on the HRS-D than those with ischaemic lesions regardless of the side of the lesion; scores were slightly higher in subjects with left-sided lesions, but the difference was not significant. However, according to the diagnostic criteria 
of the HRS-D only nine patients presented major depression in the acute phase, and four after three months.

Few studies to date have carried out longitudinal follow-up in patients with subcortical stroke and affective states (depression and hypomania). The results obtained in this sample of patients with lesions produced by subcortical stroke suggest that affective states improve considerably after three months.

The handicap of this study is the great heterogeneity of patients, by volume and location of their lesions, making it difficult to draw conclusions with greater reliability. In the future, we need carried out research with more homogeneous samples, that is able to relate the injuries to scores of tests that measure cognitive and affective behavior.

\section{REFERENCES}

[1] J. L. Cummings, "Neuropsychiatric Manifestations of Right Hemisphere Lesions," Brain and Language, Vol. 57, No. 1, 1997, pp. 22-37. doi:10.1006/brln.1997.1832

[2] J. L. Cummings and M. F. Mendez, "Secondary Mania with Focal Cerebrovascular Lesions," American Journal of Psychiatry, Vol. 141, No. 9, 1984, pp. 1084-1087.

[3] M. F. Folstein, R. Maigberger and P. R. McHugh, "Mood Disorder as Specific Complication of Stroke," Journal of Neurology, Neurosurgery \& Psychiatry, Vol. 40, No. 10, 1977, pp. 1018-1020. doi:10.1136/jnnp.40.10.1018

[4] M. R. Eastwood, S. L. Rifat, H. Nobbs, et al., "Mood Disorder Following Cerebrovascular Accident," British Journal of Psychiatry, Vol. 154, 1989, pp. 195-200. doi:10.1192/bjp.154.2.195

[5] M. Sharpe, K. Hawton, A. House, et al., "Mood Disorder in Long-Term Survivors of Stroke: Association with Brain Location and Volume," Psychological Medicine, Vol. 20, No. 4, 1990, pp. 815-828. doi:10.1017/S0033291700036503

[6] J. Kulisevsky, M. L. Berthier and J. Pujol, "Hemiballismus and Secondary Mania Following a Right Thalamic Infarction," Neurology, Vol. 43, No. 7, 1993, pp. 14221424. doi:10.1212/WNL.43.7.1422

[7] S. E. Starkstein, H. S. Mayberg, M. L. Berthier, et al., "Mania after Brain Injury: Neuroradiological and Metabolic Findings," Annals of Neurology, Vol. 27, No. 6, 1990, pp. 652-259. doi:10.1002/ana.410270612

[8] S. E. Starkstein and R. G. Robinson, "Mechanism of Disinhibition after Brain Lesions," Journal of Nervous \& Mental Disease, Vol. 185, No. 2, 1997, pp. 108-114. doi:10.1097/00005053-199702000-00007

[9] G. D. Pearlson, "Structural and Functional Brain Changes in Bipolar Disorder: A Selective Review," Schizophrenia Research, Vol. 39, No. 2, 1999, pp. 133-140. doi:10.1016/S0920-9964(99)00112-7

[10] S. C Modell, J. M. Mountz, G. Curtis, et al., "Neurophysiologic Dysfunction in Basal Ganglia/Limbic Striatal and Thalamocortical Circuits as a Pathogenetic Mechanism of Obsessive-Compulsive Disorder," Journal of
Neuropsychiatry \& Clinical Neurosciences, Vol. 1, No. 1, 1989, pp. 27-36.

[11] K. J. Swoboda and M. A. Jenike, "Frontal Abnormalities in a Patient with Obsessive-Compulsive Disorder: The Role of Structural Lesions in Obsessive-Compulsive Behavior," Neurology, Vol. 45, No. 12, 1995, pp. 2130 2134. doi:10.1212/WNL.45.12.2130

[12] R. C. Chacko, M. A. Corbin and R. G. Harper, "Acquired Obsessive-Compulsive Disorder Associated with Basal Ganglia," Journal of Neuropsychiatry and Clinical Neurosciences, Vol. 12, No. 2, 2000, pp. 269-272. doi:10.1176/appi.neuropsych.12.2.269

[13] T. Abel, G. Stein, S. Pereyra, et al., "Neuropsychological Study of 12 Patients with Pure Degenerative Cerebellar Disease," Revue Neurologique, Vol. 40, No. 8, 2005, pp. 465-472.

[14] American Psychiatric Association, "DSM-IV-TR Diagnostic and Statistical Manual of Mental Disorder," 4th Edition, American Psychiatric Publishing, Washington DC, 2000.

[15] J. L. Cummings, M. D. Mega, K. Gray, et al., "The Neuropsychiatric Inventory: Comprehensive Assessment of Psychopathology in Dementia," Neurology, Vol. 44, No. 12, 1990, pp. 2308-2314. doi:10.1212/WNL.44.12.2308

[16] M. A. Hamilton, "A Rating Scale for Depression," Journal of Neurology, Neurosurgery \& Psychiatry, Vol. 23, 1960, pp. 56-62. doi:10.1136/jnnp.23.1.56

[17] P. Bech, T. G. Bolwing, P. Kram and O. J. Rafaelsen, "The Bech-Rafaelsen Mania Scale and Hamilton Depression Scale," Acta Psychiatrica Scandinavica, Vol. 59, No. 4, 1979, pp. 420-430. doi:10.1111/j.1600-0447.1979.tb04484.x

[18] R. J. Hodgson and S. Rachman, "Obsessional-Compulsive Complaints," Behaviour Research and Therapy, Vol. 15, No. 5, 1977, pp. 389-395. doi:10.1016/0005-7967(77)90042-0

[19] M. F. Folstein, S. E. Folstein and P. R. McHugh, "'MiniMental State': A Practical Method for Grading the Cognitive State of Patients for the Clinician," Journal of Psychiatric Research, Vol. 12, No. 3, 1975, pp. 189-198. doi:10.1016/0022-3956(75)90026-6

[20] P. Gerhardt and W. Frommhold, "Atlas of Anatomic Correlations in CT and MRI," Thieme-Stratton Corp., New York, 1987.

[21] D. Sinyor, P. Jacques, D. G. Kaloupek, et al., "Poststroke Depression and Lesion Location. An Attempted Replication," Brain, Vol. 109, No. 3, 1986, pp. 537-546. doi:10.1093/brain/109.3.537

[22] S. E. Starkstein, R. G. Robinson and T. R. Price, "Comparison of Patients with and without Poststroke Major Depression Matched for Size and Location of Lesion," Archives of General Psychiatry, Vol. 45, No. 3, 1988, pp. 247-252.

[23] J. R. Lipsey, R. G. Robinson, G. D. Pearlson, et al., "Mood Change Following Bilateral Hemisphere Brain Injury," British Journal of Psychiatry, Vol. 143, 1983, pp. 266-73. doi:10.1192/bjp.143.3.266

[24] J. R. Lipsey, W. C. Spencer, P. V. Rabins, et al., "Phe- 
nomenal Comparison of Post-Stroke Depression and Functional Depression," American Journal of Psychiatry, Vol. 143, No. 4, 1986, pp. 527-529.

[25] R. G. Robinson, K. L. Kubos, L. B. Starr, et al., "Mood Disorder in Stroke Patients: Importance of Location of Lesion," Brain, Vol. 107, No. 1, 1984, pp. 81-93. doi:10.1093/brain/107.1.81

[26] A. House, M. Dennis, L. Mogridge, et al., "Mood Disorder in the Year after First Stroke," British Journal of Psychiatry, Vol. 158, 1991, pp. 83-92. doi:10.1192/bjp.158.1.83

[27] S. Signer, J. L. Cummings and D. F. Benson, "Delusions and Mood Disorder in Patients with Chronic Aphasia," Journal of Neuropsychiatry, Vol. 1, No. 1, 1989, pp. 4045.

[28] R. A. Stern and D. L. Bachman, "Depressive Symptoms Following Stroke," American Journal of Psychiatry, Vol. 148, No. 3, 1991, pp. 351-356.

[29] R. G. Robinson, J. D. Boston, S. E. Starkstein, et al., "Comparison of Mania and Depression after Brain Injury:
Causal factors," American Journal of Psychiatry, Vol. 145, No. 2, 1988, pp. 172-178.

[30] S. Simpson and B. Baldwin, "Neuropsychiatry and SPECT of an Acute Obsessive-Compulsive Syndrome Patient," British Journal of Psychiatry, Vol. 166, No. 3, 1995, pp. 390-392. doi:10.1192/bjp.166.3.390

[31] J. L. Cumming and J. I. Victoroff, "Noncognitive Neuropsychiatric Syndromes in Alzheimer's Disease," Neuropsychiatry, Neuropsychology \& Behavioral Neurology, Vol. 3, No. 2, 1990, pp. 140-158.

[32] J. L. Cummings, B. Miller, M. A. Hill, et al., "Neuropsychiatric Aspects of Multi-Infarct Dementia and Dementia of the Alzheimer Type," Archives of Neurology, Vol. 44, No. 4, 1987, pp. 389-393.

[33] P. Birkett, "The Psychiatry of Stroke," American Psychiatric Press, Washington DC, 1966.

[34] A. E. Hillis, R. J. Wityk, P. B. Barker, et al., "Subcortical Aphasia and Neglect in Acute Stroke: The Role of Cortical Hypoperfusion," Brain, Vol. 125, No. 5, 2002, pp. 1094-1104. doi:10.1093/brain/awf113 